\title{
Glycol Chitosan-Based Fluorescent Theranostic Nanoagents for Cancer Therapy
}

\section{Jin-Kyu Rhee ${ }^{1, \dagger}$, Ok Kyu Park ${ }^{2, \dagger}$, Aeju Lee ${ }^{3}$, Dae Hyeok Yang ${ }^{4, *}$ and Kyeongsoon Park ${ }^{2, *}$}

1 Western Seoul Center, Korea Basic Science Institute, Seoul 120-140, Korea; E-Mail: jkrhee@kbsi.re.kr

2 Division of Bio-imaging, Chuncheon Center, Korea Basic Science Institute, Gangwon-do 200-701, Korea; E-Mail: okpark81@kbsi.re.kr

3 Biomedical Research Center, Korea Institute of Science and Technology, Seoul 136-791, Korea; E-Mail: fleur0903@hanmail.net

4 Institute of Cell \& Tissue Engineering, College of Medicine, The Catholic University of Korea, Seoul 137-701, Korea

$\dagger$ These authors contributed equally to this work.

* Authors to whom correspondence should be addressed; E-Mails: kspark1223@hanmail.net (K.P.); yangdh@catholic.ac.kr (D.H.Y.); Tel.: +82-33-815-4604 (K.P.); +82-2-2258-7497 (D.H.Y.); Fax: +82-33-255-7273 (K.P.); +82-2-2258-7494 (D.H.Y.).

External Editor: David Harding

Received: 9 October 2014; in revised form: 27 November 2014 / Accepted: 27 November 2014 / Published: 17 December 2014

\begin{abstract}
Theranostics is an integrated nanosystem that combines therapeutics with diagnostics in attempt to develop new personalized treatments with enhanced therapeutic efficacy and safety. As a promising therapeutic paradigm with cutting-edge technologies, theranostic agents are able to simultaneously deliver therapeutic drugs and diagnostic imaging agents and also monitor the response to therapy. Polymeric nanosystems have been intensively explored for biomedical applications to diagnose and treat various cancers. In recent years, glycol chitosan-based nanoagents have been developed as dual-purpose materials for simultaneous diagnosis and therapy. They have shown great potential in cancer therapies, such as chemotherapeutics and nucleic acid and photodynamic therapies. In this review, we summarize the recent progress and potential applications of glycol chitosan-based fluorescent theranostic nanoagents for cancer treatments and discuss their possible underlying mechanisms.
\end{abstract}


Keywords: glycol chitosan; theranostics; nanosystem; fluorescence; cancer

\section{Introduction}

Theranostics is a system that integrates targeting, therapeutic, and diagnostic functions within an all-in-one platform [1]. Currently, diagnostic imaging and treatment processes are carried out separately in the study of the characteristics (i.e., cellular phenotype, heterogenecity, etc.) of cancers and administration of drugs for therapy [2,3]. These separate processes require a long time period in which to evaluate drug efficacy and adjust the treatment plan accordingly, resulting in loss of opportunity for effectively treating some diseases, especially rapidly progressing cancers [4]. In contrast, integrated theranostic nanoagents can deliver diagnostic imaging agents capable of detecting and monitoring the early onset of diseases and simultaneously transport suitable therapeutic drugs over a prolonged period in order to enhance therapeutic efficacy [5]. This all-in-one theranostic approach is less time consuming and subsequently allows for a faster and more precise decision, allowing for more effective outcomes. Furthermore, theranostic technologies will radically change the way we diagnose, treat, and prevent cancer in oncology, and they show great promise in the emerging field of personalized medicine.

Advances in nanotechnology have contributed to the development of novel multifunctional nanoagents that enable specific delivery of imaging agents and therapeutic drugs to target diseased tissues for cancer imaging and therapy, and thus, they have ultimately led to the newest technology, "theranostics." Nanotheranostics involves the application and further development of various nanoparticle systems, such as polymer conjugations, dendrimers, micelles, liposomes, metal and inorganic nanoparticles, carbon nanotubes, and polymeric nanoparticles, for sustained, controlled, and targeted co-delivery of diagnostic and therapeutic agents in order to achieve improved results and fewer side effects [6]. Since these nanoparticles have nanoscale size dimensions (10-500 nm), they can navigate through microvasculatures and across various biological barriers to preferentially accumulate in tumor tissues due to the "enhanced permeability and retention" (EPR) effects, which are the hallmark of leaky vasculatures and poor lymphatic drainage [7]. Ample functional groups and easy modification with hydrophobic segments allow these nanoparticles to be modified by imaging agents and therapeutic drugs, which can either be encapsulated by or conjugated to polymeric nanoparticles. Therefore, polymeric nanoparticles have been employed to serve as diagnostic tools, therapeutic carriers, or both [4]. Additionally, polymeric nanoparticles improve the half-lives, solubility, and stability of imaging probes and therapeutic drugs while dramatically reducing potential side effects [8-10]. These features make polymeric nanoparticles preferable for customized and personalized translational medicine.

A diverse set of non-invasive imaging modalities is employed for the detection of cancer at early stages, discovery and development of new drugs, and monitoring of drug responses by offering information about biological changes and living systems, as well as visualization of the distribution of theranostic nanoagents in real time $[4,11,12]$. These non-invasive imaging modalities include positron-emission tomography (PET), magnetic resonance imaging (MRI), X-ray computed tomography (CT), single photon emission computed tomography (SPECT), ultrasound (US), and 
optical fluorescence imaging [12]. As shown in Figure 1, each imaging modality has its own unique advantages and disadvantages regarding sensitivity, spatial resolution, cost, safety, and tissue penetration [13]. Recently, near-infrared fluorescence (NIRF) imaging techniques have been used for real-time imaging in live animals. Although poor tissue penetration must be overcome with this method when applied in clinics, the NIRF imaging system is safe, highly sensitive, and capable of multicolor imaging to monitor the fate of theranostic agents in live animals without the requirement of a local cyclotron, incontinent radionuclide-labeling step, or expensive instruments [14]. Also, an optical imaging approach in the NIR window $(700-900 \mathrm{~nm})$ is very useful to identify the fundamental processes at the cellular and molecular levels because the absorbance and autofluorescence of hemoglobin (the main absorber in the visible region), water, and lipids (primary absorbers of infrared light) are lower in the NIR range [15].

Figure 1. Non-invasive imaging modalities and their characteristics for biomedical applications. Permission from reference [13], Copyright (C) 2011, Royal Society of Chemistry.

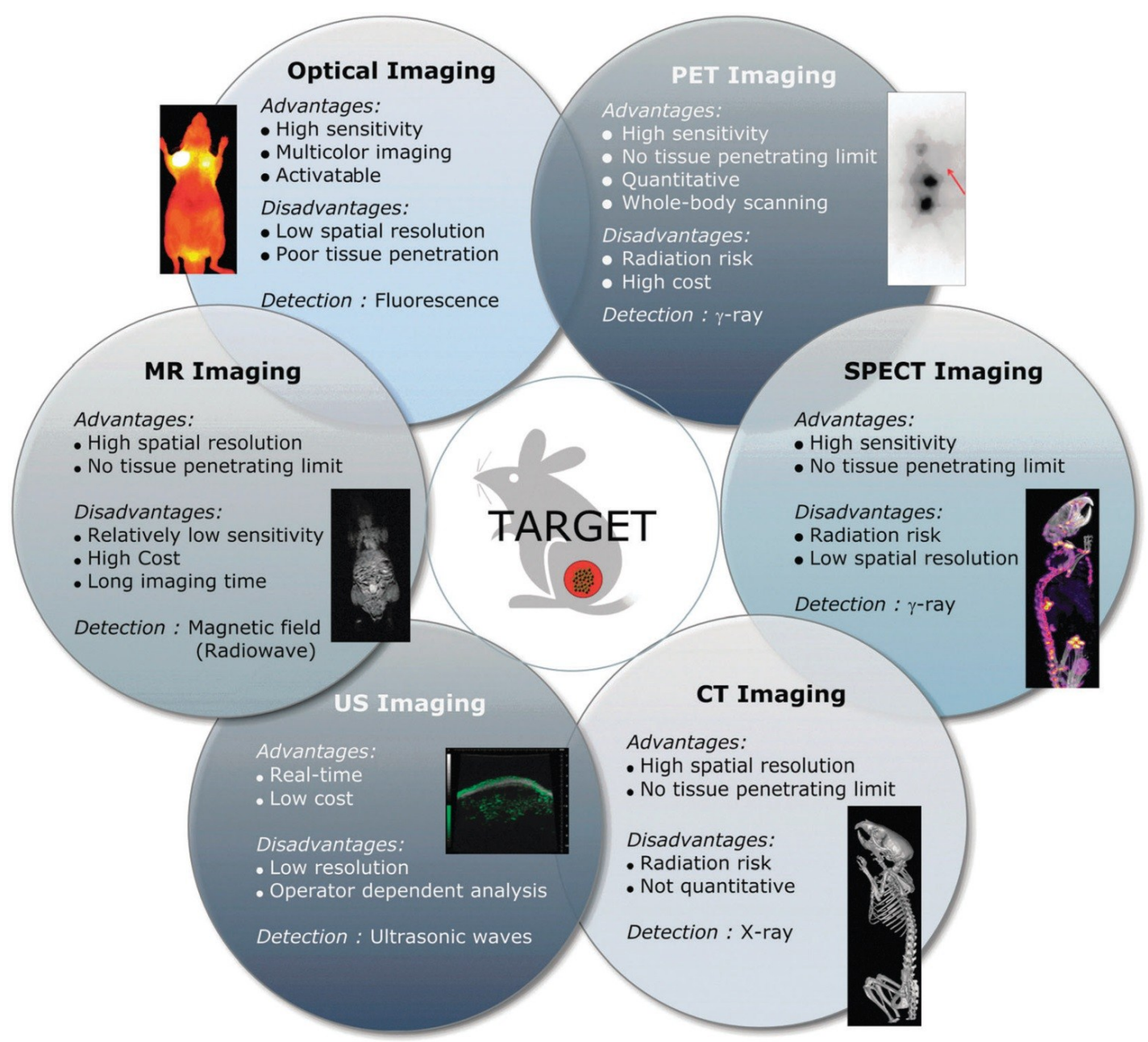

Chitosan, a deacetylated derivative of chitin, is a copolymer that consists of two repeating units (i.e., $\quad N$-acetyl-2-amino-2-D-glucopyranose and 2-amino-2-deoxy-D-glucopyranose) linked by a $\beta$-( $1 \rightarrow 4)$-glycosidic bond [16]. Chitosan is a biocompatible, biodegradable, and low-immogenic polymer that has great potential for a wide range of biomedical applications, such as drug delivery, gene delivery, cell imaging, and as a sensor in the treatment and diagnosis of some diseases [16]. However, the poor 
solubility of chitosan in aqueous solutions at $\mathrm{pH}>6.0$ is a major limitation to these potential uses. Glycol chitosan (GC) is a water-soluble chitosan derivative at neutral $\mathrm{pH}$ due to the introduction of a hydrophilic ethylene glycol group. Over the last 10 years, GC-based polymeric nanoparticles have been developed as therapeutic carriers (i.e., anticancer drugs [17-19], peptides [20,21], nucleic acids [22,23], and diagnostic imaging agents [24-26]) in oncology. This review article describes the recent progress with GC-based fluorescent theranostic nanoagents as dual-purpose agents used for simultaneous diagnosis and therapy and further discusses their possible underlying mechanisms.

\section{Basic Components for Manufacturing Theranostic Nanoagents}

Integrated theranostic nanoagents offer multifunctional platforms for simultaneous cancer diagnosis and therapy. They provide a great deal of information for rapid disease verification and exact localization of the diseased tissues and permit the rapid delivery of therapeutic agents to the target tissues. To achieve the concept of theranostics, most theranostic nanoagents are typically designed in consideration of four basic components: signal emitter, therapeutic payload, payload carrier, and targeting ligand [4]. Representative examples of the components are shown in Figure 2 and Table 1.

Figure 2. Four basic components for the design of theranostic nanoagents.

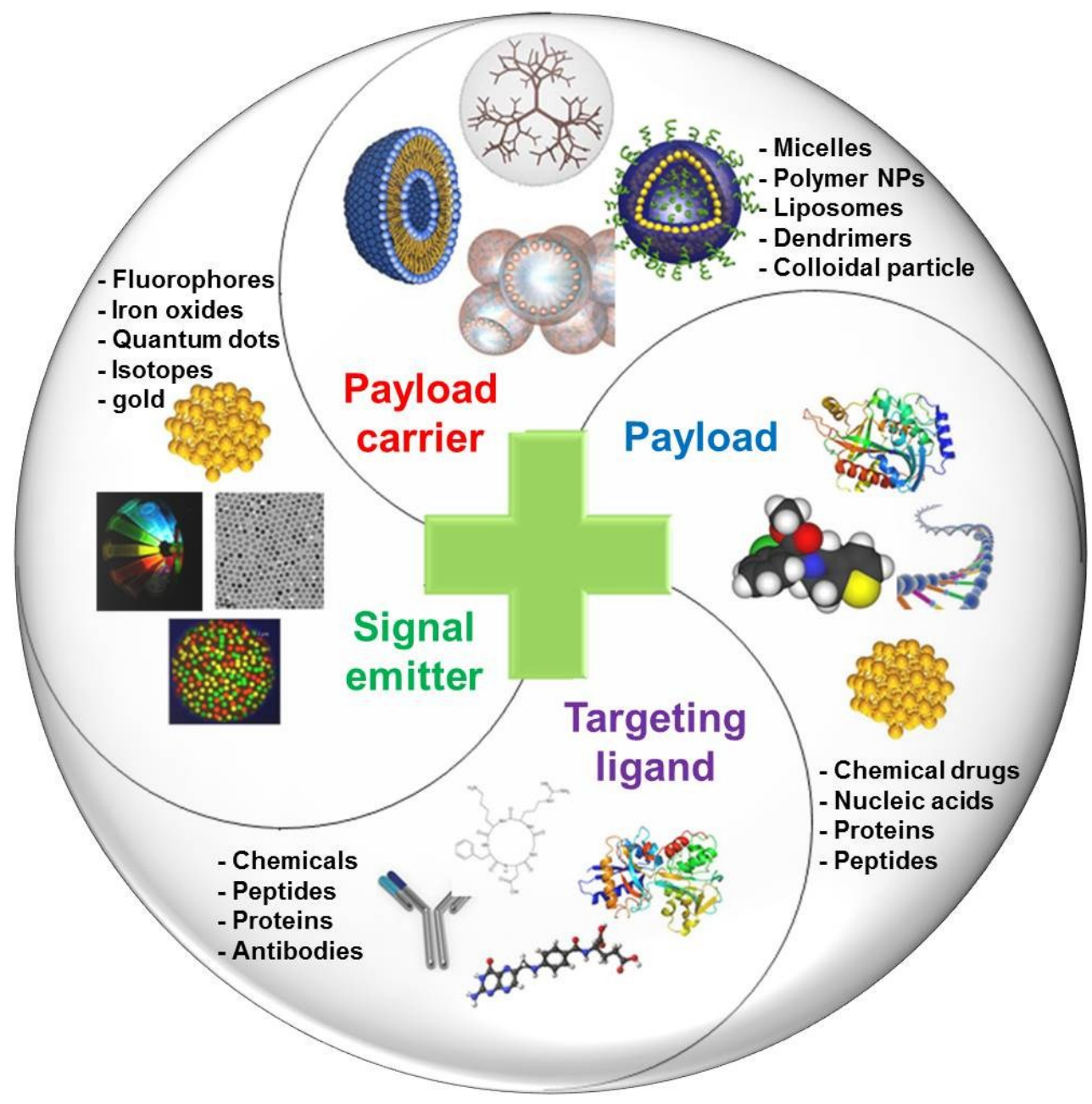


Table 1. Examples of four basic components for the design of theranostic nanoagents.

\begin{tabular}{ll}
\hline Components & \multicolumn{1}{c}{ Examples } \\
\hline & Fluorophores: Fluorescein isothiocyanate (FITC), Alexa Fluor 488, yellow \\
& fuorescent protein (YFP), Rhodamine, tetramethylrhodamine (TRITC), cyanine \\
& 3(Cy3), red fluorescent protein (RFP), Texas Red, Cy5, Alexa Fluor 647, Cy5.5, \\
Signal Emitters & Cy7, protoporphyrin IX (PpIX), and chlorine e6 $(\mathrm{Ce} 6)$, etc. \\
& Isotopes: ${ }^{76} \mathrm{Br},{ }^{124} \mathrm{I},{ }^{94 \mathrm{~m}} \mathrm{Tc},{ }^{68} \mathrm{Ga},{ }^{66} \mathrm{Ga},{ }^{60} \mathrm{Cu},{ }^{64} \mathrm{Cu},{ }^{89} \mathrm{Zr}$, etc. \\
& Magnetic resonance imaging agents: iron oxide, iron platinum, \\
& manganese, gadolinium, etc. \\
& Other metals: Quantom dots, silver and gold nanoparticles, etc. \\
& Chemical drugs: Doxorubicin, cisplatin, paclitaxel, docetaxel, \\
& camptothecin, mitoxantrone, gemcitabin, curcumin, photosensitizers, \\
& imatinib, trastuzumab, sinutinib, cetuximab, etc. \\
& Protein and peptide drugs: RGD, buserelin, gonadorelin, leuprolide, triptorelin, \\
& abarelix, cetrorelix, Tumor necrosis factor-related apoptosis-inducing ligand \\
Payload & (TRAIL), melanoma differentiation associated-7 (MDA-7), E1A, E4ORF4, VP3 \\
& (apoptin), cytokines (interleukin-2, interferon alpha-n3, interferon beta-1, \\
& inteferon alpha-2b), monoclonal antibodies (zevalin, mylotarg, \\
& bexxar, herceptin, avastin), etc. \\
& Neucleic acids: Plasmids, antisense oligonucleotides, ribozymes, \\
& DNAzymes, aptamers, small interfering RNA (siRNA), \\
& small hairpin RNA (shRNA), and microRNA, etc. \\
\hline \multirow{2}{*}{ Payload } & Macromolecular prodrugs, stealth nanoparticles, micelles, nanogels, \\
& nanocapsules, polymersomes, liposomes, dendrimers, porous \\
& silica nanoparticles, etc. \\
\hline & Angiogenic endothelial cell target: RGD, vascular endothelial \\
growth factor, TGN peptide, etc. \\
Cancer cell target: Epidermal growth factor receptor monoclonal antibody, \\
epidermal growth factor, human epidermal receptor 2, transferrin, A10 aptamer, \\
As1411 aptamer, cRGD, galactose, hyaluronic acid, folic acid, glycyrrhizin, etc. \\
\hline
\end{tabular}

The signal emitter possesses certain unique optical, magnetic, or radioactive properties. Therapeutic drugs can be chemotherapeutic drugs, therapeutic proteins and peptides, or nucleic acids. The drug carrier is a kind of matrix comprised of polymeric materials. In general, intact and modified polymers have ample functional groups ( such as $-\mathrm{NH}_{2},-\mathrm{COOH},-\mathrm{OH},-\mathrm{SH}$, or $-\mathrm{N}_{3}$, etc.) that offer flexibility in integrating multifunctionalities. Due to this flexibility, both the signal emitter and therapeutic drugs can be either non-covalently encapsulated in the carrier via hydrophobic and/or electrostatic interactions or covalently conjugated to the surface of the drug carrier [27,28]. Importantly, most of the synthetic and natural polymers used for biomedical applications are cleavable and biodegradable. Sometimes, the unique characteristics of tumor microenvironments, such as enzymes and $\mathrm{pH}$, which would trigger drug release from a drug carrier, are used as another drug delivery approach. For example, enzymatic cleavage is highly specific for certain tumors where specific enzymes are overexpressed. Enzymatically-degradable drug delivery carriers showed a rapid drug release upon specific enzyme exposure, whereas minimal drug release occurs without enzymes. In case of non-enzymatic drug carriers, they showed the sustained delivery of therapeutic drugs at the target site. 
Finally, targeting ligands with high affinity to tumor cells or angiogenic endothelial cells are always covalently attached to the surface of the drug carriers [29]. Because passive targeting delivery of nanoparticles does not often operate effectively in poorly vascularized regions, an active targeting approach using a targeting ligand is required in order to increase accumulation of the nanoparticle in the disease tissue and to facilitate detection efficiency while reducing unwanted uptake by normal tissue, thus minimizing side effects. As described above, to meet the requirements for theranostics, the manufacturing processes of multifunctional theranostic nanoagents as a single system must be fairly complicated. However, theranostic nanoagents have promising advantages because they allow in situ imaging, targeted delivery, and controlled release of therapeutic drugs to diseases sites, as well as real-time monitoring of treatment responses.

\section{Glycol Chitosan-Based Theranostic Nanoagents}

A variety of polymeric nanoparticles for drug delivery, diagnosis, and therapy have been developed. Among them, glycol chitosan (GC) has attracted interest because it is soluble in aqueous media, biocompatible, biodegradable, and has low immunogenicity [30]. Over the last 10 years, GC-based nanoparticles (GC NPs) have been intensively studied as drug delivery carriers for therapy and/or imaging agents for diagnosis. GC NPs are prepared by chemical conjugation of a hydrophilic GC polymer $(250 \mathrm{kDa})$ with hydrophobic molecules. At the initial stage, GC NPs were developed as drug delivery carriers for cancer therapy. Due to their hydrophobic moieties and positive charge, GC NPs can non-covalently encapsulate hydrophobic cancer drugs [17-19], peptides [20], or nucleic acids [22] and still show potent therapeutic efficacy. Since 2006 when various non-invasive optical imaging modalities became widespread, optical imaging probes for the detection of various cancers (head and neck, brain, liver, colon, and metastatic cancer) were also developed by introducing a near-infrared fluorophore (Cyanine 5.5, Cy5.5) to GC NPs [24,26,31]. The constructed Cy5.5-labeled GC-5 $\beta$-cholanic acid conjugates formed self-assembled nanostructures with diameters of $260 \pm 30 \mathrm{~nm}$ and showed good colloidal stability for up to one month in PBS (pH 7.4) [24] (Figure 3A,B). To verify the superior tumor-targeting efficacy of Cy5.5-labeled GC NPs, several key factors influencing biodistribution and tumor-targeting of the nanoparticle system were investigated. Firstly, Cy5.5 (small molecule), Cy5.5-labeled GC (soluble polymer), and Cy5.5-labeled GC NPs were intravenously injected to SCC7 (squamous cell carcinoma) tumor-bearing mice. Compared to fluorescence intensities of Cy5.5 and Cy5.5-labeled GC, Cy5.5-labeled GC NPs exhibited strong fluorescence signals in the whole body and in tumor tissues for up to three days (Figure 3C), implying that the nanoparticle system has much stronger tumor-targeting characteristics than do small molecules and soluble polymer. Secondly, whole body fluorescence images of three types of Cy5.5-labeled GC NPs using different molecular weights of GC $(20,100$, and $250 \mathrm{kDa})$ were performed to investigate the effects of molecular weight on tumor-targeting efficacy [24]. In vivo fluorescence images showed that the highest molecular weight GC NPs had a much longer circulation time in the blood stream and were more preferentially accumulated in tumor tissues than were the relatively lower molecular weight GC NPs. Thirdly, the deformability of GC NPs is also an important factor influencing tumor-targeting efficiency. As red blood cells $(8 \mu \mathrm{m})$ of a highly flexible nature can readily pass through small micro-vessels $(2.5 \mu \mathrm{m})$ [32], more than $95 \%$ of Cy5.5-labeled GC NPs $(260 \pm 30 \mathrm{~nm})$ easily passed through a $200-\mathrm{nm}$ pore size filter. 
However, 200-nm rigid Cy5.5-labeled polystyrene (PS) NPs did not easily pass through even a 450-nm pore size filter (Figure 3D). Indeed, deformable Cy5.5-labeled GC NPs showed strong NIRF signals in tumor tissues after passing through the in vivo filtration system of the liver and spleen [31] (Figure 3E). However, rigid and non-deformable PS NPs were primarily localized in the liver and spleen. These results suggest that the particles must be either smaller than the critical size or deformable enough to avoid in vivo filtration systems [33]. Finally, GC NPs have a positive surface charge and lipophilicity to facilitate rapid cellular uptake, which may improve the residence time of NPs in tumor tissues and thereby enhance the efficiency of diagnosis and therapy. These properties demonstrate the excellent tumor-targeting characteristics of GC-based theranostic NPs, making them one of the more promising of the recently developed GC-based fluorescent theranostic nanoagents that are being studied for simultaneous diagnosis and therapy.

Figure 3. (A) Chemical structure of Cy5.5-labeled glycol chitosan-5 $\beta$ cholanic acid conjugates and TEM image; (B) Colloidal stability of Cy5.5-labeled GC NPs in PBS for one month; (C) Time-dependent tumor targeting specificity of free Cy5.5, water soluble Cy5.5-labeled glycol chitosan polymer, and Cy5.5-labeled GC NPs in SCC7 tumor-bearing mice; (D) Filtration test of Cy5.5-labeled glycol chitosan polymer, Cy5.5-labeled GC NPs, and Cy5.5-labeled polystyrene beads with different pore sizes; (E) Ex vivo organ distribution of (a) Cy5.5-labeled GC NPs and (b) Cy5.5-labeled polystyrene beads. Reprinted with permission from [31] and [33], Copyright (C) 2011 and 2010 Elsevier.

A

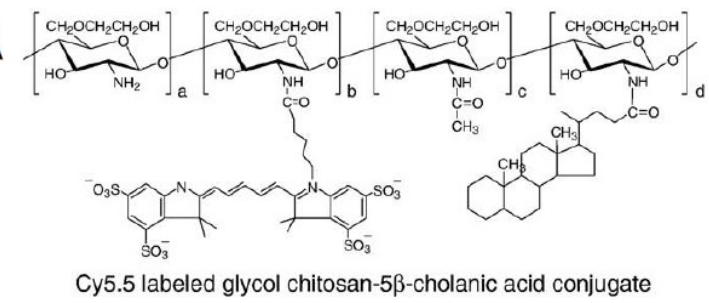

B

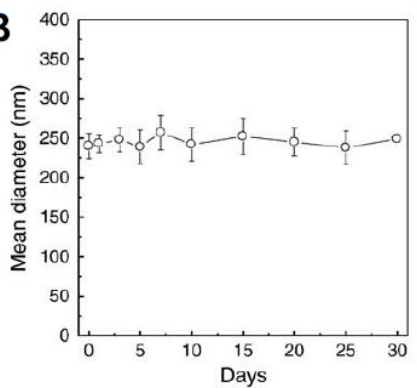

D

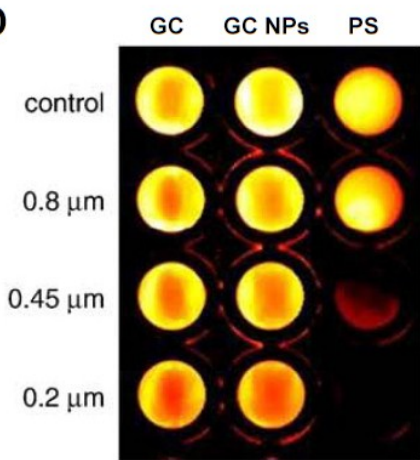

C

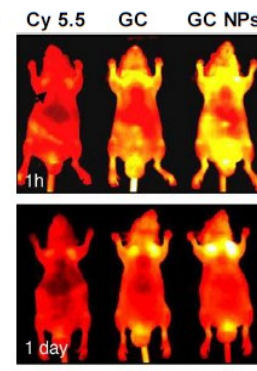

E

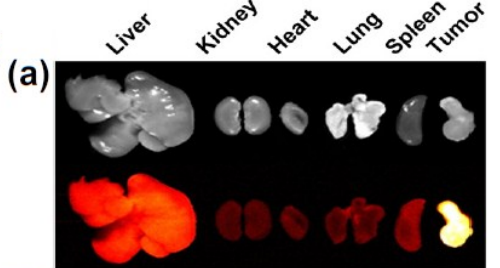

(b)
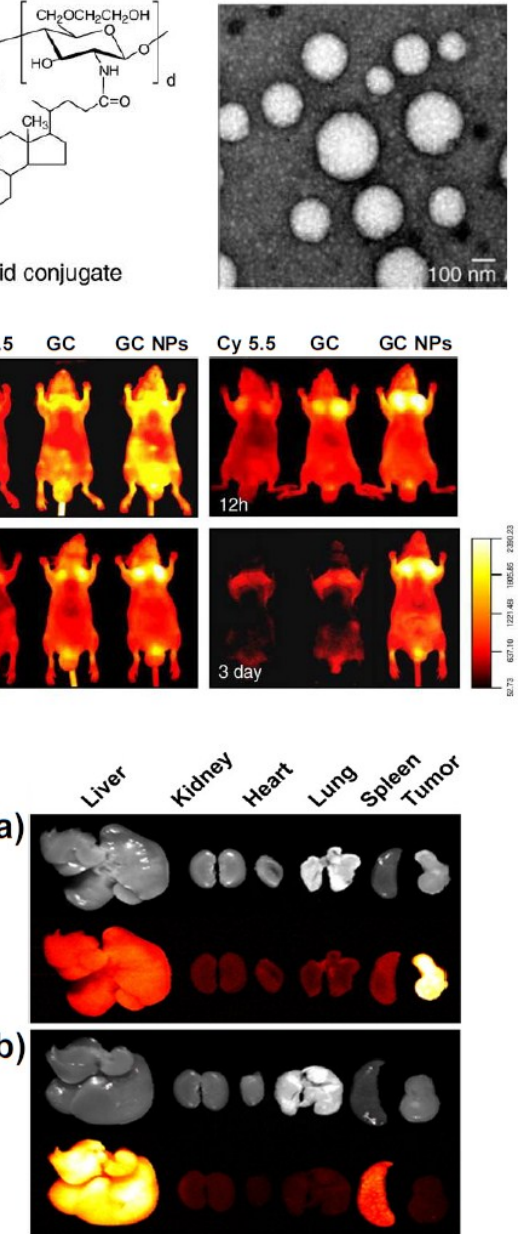


\subsection{Small Molecular Drug-Based Theranostic Nanoagents}

As previously described, GC NPs have good colloidal stability, deformability (or flexibility), and rapid cellular uptake and are very suitable as drug delivery carriers and imaging agents. To achieve the theranostic concept in an all-in-one platform, chemotherapeutic drugs are non-covalently encapsulated in Cy5.5-labeled GC NPs. Here, Cy5.5, GC NPs, and chemotherapeutic drugs act as signal emitter, payload carrier, and payload, respectively. Using several Cy5.5-labeled GC NPs constructed by conjugation of GC with 5 $\beta$-cholanic acid, deoxycholic acid, or hydrotropic oligomer, chemotherapeutic drugs, such as paclitaxel [33,34], cisplatin [35], camptothecin [36], or docetaxel [37], can be readily encapsulated into the GC NPs via hydrophobic interactions. These GC NPs can be used as a Cremophor EL (polyoxyethylated caster oil)/absolute ethanol-free formulation to solubilize extremely insoluble drugs, such as paclitaxel [33,34] and docetaxel [37]. The GC NP system can also protect the drug activity of hydrolysis-labile drugs, such as camptothecin [36]. Furthermore, hydrotropic DENA (N,N-diethylnicotinamide)-based polymeric GC NPs can enhance drug-loading content (or efficiency) by almost two-fold compared to that of GC-5 $\beta$-cholanic acid NPs because hydrotropic oligomers as water-soluble compounds enhance the water solubility of sparingly soluble drugs [34,38]. With the help of in vivo fluorescence imaging modalities, several pieces of information were confirmed: Cy5.5-labeled GC NPs containing chemotherapeutic drugs have a long circulation time (three to seven days), show specific tumor accumulation (nearly two- to four-fold) compared to that in normal tissues [33,34,37], and exhibit dose-dependent delivery efficiency to tumors. Also, the repeated injection frequency (or intervals) of theranostic nanoagents can be determined by monitoring the NIRF signal in tumor tissues. Therefore, based on the obtained important information driven by NIRF whole and ex vivo images, the therapeutic efficacies of the nanoagents can be greatly maximized. This evidence suggests that the use of theranostic nanoagents will play an important role in attaining more specific information, such as circulation time, biodistribution, nanoparticle injection intervals and dosages, and therapeutic response monitoring, allowing for optimized treatment protocols.

Typically, when researchers develop theranostic nanoagents, they make an effort to produce optimized systems with high targeting efficiency. However, bare nanoparticles and drug-loaded nanoparticles have quite different biodistributions and tumor-targeting characteristics [39]. In fact, the in vivo fate of a nanoparticle system can be altered because the physicochemical properties of nanoparticle systems, such as particle size, colloidal stability, and deformability, can be different before and after drug encapsulation into nanoparticle systems. Therefore, researchers should perform in vivo studies with a single platform containing both signal emitter and payload in order to obtain more accurate and precise information regarding theranostic nanosystems.

\section{2. siRNA-Based Theranostic Glycol Chitosan Nanoagents}

Nucleic acid therapy is promising for the treatment of various diseases, such as Alzheimer's disease, cancer, adenosine deaminase deficiency, and cystic fibrosis [40]. Current and potential therapeutic nucleic acids include plasmids, antisense oligonucleotides, ribozymes, DNAzymes, aptamers, and small interfering RNA (siRNA). These therapeutic nucleic acids can alter gene expression at the transcriptional or post-transcriptional level and may be effective in treating cancer 
and cardiovascular and inflammatory diseases. Although they are powerful therapeutic agents for the treatment of diseases, there are still obstacles to be overcome. Naked nucleic acids are highly susceptible to enzymatic degradation. Also, large and negatively-charged nucleic acids are not easily internalized by cells due to the difficult in crossing negatively-charged cell membranes. Thus, naked nucleic acid delivery requires direct injection of nucleic acids to the disease areas via physical methods, such as electroporation, gene gun, or ultrasound [41]. However, systemic delivery of naked nucleic acids is quite ineffective due to the minimal amount of intact polynucleotide reaching diseased sites because of the degradation by nucleases within the physiological fluids. Also, the direct injection approach limits efficient delivery only to anatomically accessible target sites.

Recently, among various nucleic acids, siRNA has attracted a great deal of attention as a potential therapeutic agent due to its highly sequence-specific gene silencing ability and generality in therapeutic targets. The difficulty of systemic siRNA is that its delivery is severely hindered in clinical applications due to its susceptibility to degradation by nuclease. To achieve efficient systemic delivery of therapeutic siRNA, many researchers have focused on the development of nonviral vehicles, which are nontoxic and less immunostimulatory and can have more versatile modifications for carrying different types of drug payloads. GC NPs have been tested for systemic delivery of siRNA; however, the electrostatic interaction between positively-charged GC NPs and negatively-charged siRNA is too weak to form condensed and stable siRNA/GC NP vehicles. To obtain vehicles that are more

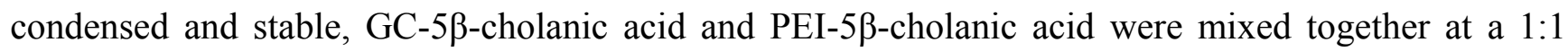
weight ratio to yield GC-PEI NPs [23]. RFP-siRNA and GC-PEI NPs at a weight ratio of 1:5 formed more condensed and stable nano complexes $(250 \mathrm{~nm})$ with nearly 100\% loading efficiency due to the increased positive charge caused by mixing GC NPs with PEI NPs. The RFP-siRNA/GC-PEI NPs successfully protected encapsulated RFP-siRNA from degradation by RNase A. Also, GC-PEI NPs effectively delivered RFP-siRNA to the cell cytoplasm and exerted remarkable silencing effects by suppressing RFP expression in RFP-expressing B16F10 cells. In vivo fluorescence images revealed that the intravenously injected Cy5.5-labeled RFP-siRNA/GC-PEI NPs were highly accumulated in tumor tissues. Due to effective delivery of RFP-siRNA into tumor tissues, the RFP signal intensity of RFP-B16F10 tumors treated with RFP-siRNA/GC-PEI NPs was 4.8-fold lower than that of the free siRNA group.

Kim and his colleagues synthesized a novel alternative siRNA, polymerized siRNA (poly-siRNA), to enhance the stability of the siRNA [42]. To polymerize siRNA, dithiol-modified siRNA (RFP-or VEGF-specific siRNA) bearing thiol groups at the 5'-ends of both sense and anti-sense strands were reacted in the presence of $N, N, N^{\prime}, N^{\prime}$-tetramethyl-azodicarboxamide (Figure 4A). The polymerized siRNA consisted of a broad range of base pairs (50-300 bps and greater than $300 \mathrm{bps}$ ) through disulfide-polymerization of thiol groups at both ends of the modified siRNA. This polymerized siRNA can be converted to original mono-siRNA in the presence of a reducing agent, such as dithiothreitol (DTT) or under reductive conditions as exist in the cell cytosol. The in vitro serum stability test showed that mono-siRNA was degraded within $1 \mathrm{~h}$, whereas most of the poly-siRNA was degraded within $12 \mathrm{~h}$, suggesting that poly-siRNA is much more stable and less degradable by serum nuclease compared to mono-siRNA [43]. Although poly-siRNA is more stable than mono-siRNA, the degradation of poly-siRNA is still very susceptible to enzymatic degradation in in vivo physiological fluids. To overcome this problem, Kim and his colleagues synthesized a new systemic 
poly-siRNA delivery carrier, thiolated GC (tGC) polymer, using sulfosuccinimidyl-6-[3' (2-pyridyldithio)-propionamido] hexanoate (Sulfo-LC-SPDP) and DTT (Figure 4B) [43]. The tGC polymer formed stable nanostructures with poly-siRNA (RFP or VEGF) through enhanced electrostatic interaction and a self-crosslinking mechanism, leading to increased serum stability up to $24 \mathrm{~h}$. The poly-siRNA/tGC (psi-tGC) NPs rapidly internalized and localized in the cytosol within $1 \mathrm{~h}$ and then showed efficient RFP gene silencing. After intravenous injection, FPR 675-labeled poly-siRNA-tGC was preferentially accumulated in tumor tissues compared to naked FPR 675-labeled poly-siRNA or FPR 675-labeled-psi/PEI polyplexes, suggesting higher tumor selectivity of poly-siRNA-tGC NPs (Figure 4C). In vivo fluorescence images and therapeutic tests demonstrated that poly-siRNA(RFP)-tGC showed effective RFP gene silencing in vivo, and poly-siRNA(VEGF)-tGC NPs also significantly inhibited neovascularization via effective and specific VEGF gene silencing, leading to successful tumor suppression (Figure 4D,E). These results support the theory that the modified GC derivatives are suitable vehicles for siRNA delivery. However, more optimized treatment protocols for safe and effective systemic siRNA delivery carriers are still required for a wide range of clinical applications of siRNA.

Figure 4. (A) Scheme of polymerized siRNA (poly-siRNA) synthesis. Reprinted with permission from [42], Copyright (C) 2009 Elsevier B.V.; (B) Preparation of poly-siRNA/tGC nanoparticles for siRNA delivery; (C) In vivo real-time NIRF imaging of poly-siRNA/tGC in SCC7 tumor-bearing mice after i.v. injection of FPR675-labeled nanoparticles; (D) Inhibition of blood vessel formation by poly-siRNA (VEGF)/tGC; (E) Antitumor effects of control, poly-siRNA, poly-siRNA (scramble)/tGC, and poly-siRNA (VEGF)/tGC. Reprinted with permission from [43], Copyright (C) 2012 WILEY-VCH Verlag.

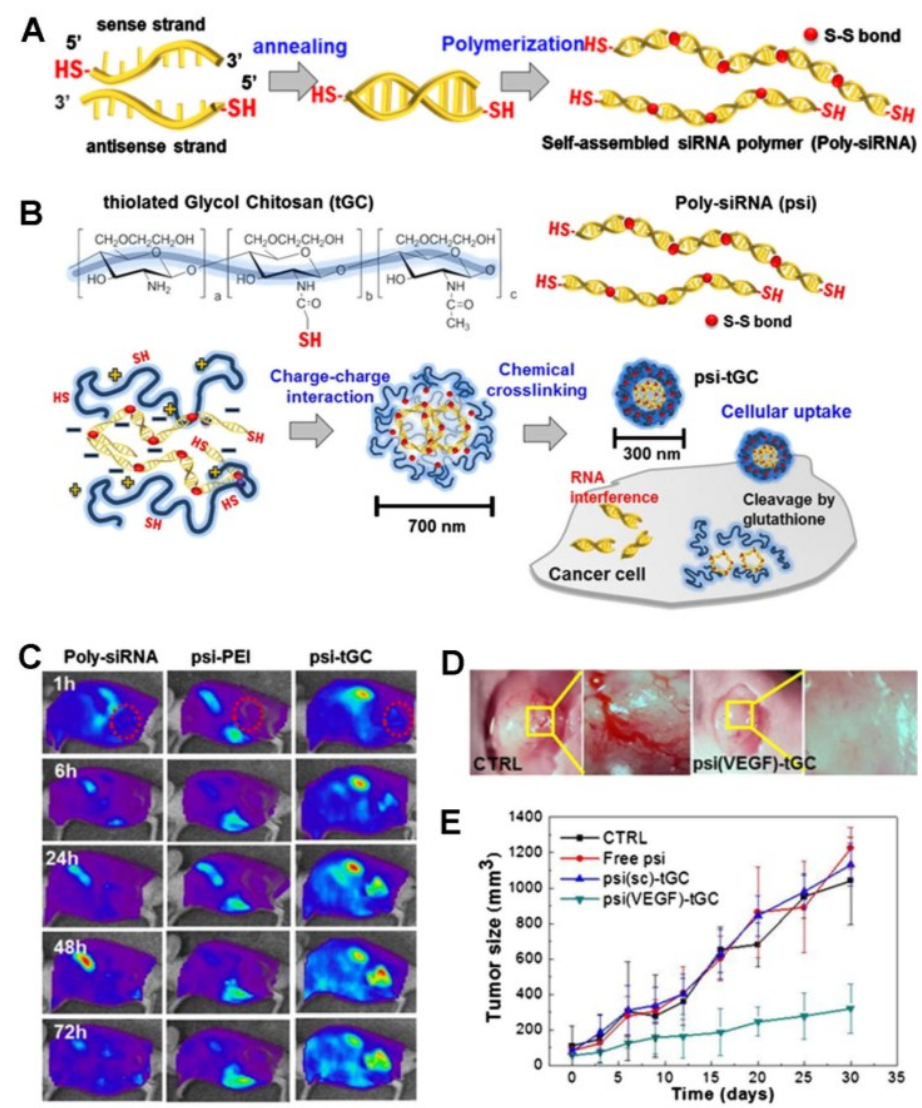




\subsection{Photosensitizer-Based Theranostic Nanoagents}

Photodynamic therapy (PDT) is a relatively new type of treatment that is attracting interest as a potential cancer treatment. PDT is a nonthermal photochemical reaction, which requires the simultaneous presence of a photosensitizing drug, oxygen, and a special type of light [44]. The photosensitizing drug alone is harmless in the absence of light and oxygen. The administered photosensitizing drug is only activated by irradiation of light to generate reactive oxygen species (ROS), including singlet oxygen $\left({ }^{1} \mathrm{O}_{2}\right)$, hydroxyl radicals $(\cdot \mathrm{OH})$, and superoxide $\left(\mathrm{O}^{2-}\right)$, which subsequently trigger lethal oxidative stress and membrane damage in the treated cells, resulting in apoptosis, necrosis, or autophagy at the area of light exposure [44-48]. In addition, photosensitizing drugs show unique luminescent properties that can be useful as fluorescence imaging agents to track, visualize, and quantify the photosensitizing drugs in diseased tissues [48]. These dual features of PS, including signal emission and payload (drug) delivery, allow photosensitizing agents to act as theranostic agents for simultaneous diagnosis and image-guided therapy.

Currently, porphyrins and their derivatives are widely used as photosensitizers (PSs) in PDT. Some of these substances are already approved for use as drugs in malignant [49] or nonmalignant disease therapies [50]. However, many first- and second-generation PSs are limited in animal studies and clinical use because of non-specific skin phototoxicity, poor water solubility at physiological $\mathrm{pH}$, and inefficient delivery to target tumor tissues in cancer treatment $[48,51,52]$. However, these limitations can be overcome by using nanoscale drug carriers. Hydrophobic PS can be encapsulated in nanoparticles or directly chemically conjugated with water-soluble polymers to enhance solubility and dispersion in an aqueous solution. Also, PS-loaded or -conjugated nanoparticles are more preferentially accumulated in tumor tissues through EPR effects [53]. The Kwon group developed protoporphyrin IX (PpIX)- and chlorin e6 (Ce6)-loaded GC NPs that are well dispersed in aqueous solution and form stable nano-structures with an average diameter around $300 \mathrm{~nm}$ [54,55]. These NPs also showed time-dependent release of PpIX or Ce6 from GC NPs and efficient photodynamic therapy in in vitro and in vivo studies. However, drugs physically loaded in nanoparticles showed burst drug release from nanoparticles during circulation in vivo [54,55]. This undesirable instability of PpIX- or Ce6-loaded GC NPs resulted in low drug delivery efficiency and decreased therapeutic effect at tumor sites as well as unintended damage to normal tissues [56]. These problems of PS-loaded nanoparticles can be overcome by chemical conjugation of PpIX or Ce6 with water soluble GC. PpIX- or Ce6-conjugated GC also formed stable and self-assembled nanoscale particles (about 250-300 nm) [55,57]. Because PpIX- or Ce6-conjugated GC NPs did not exhibit burst release from NPs due to chemical conjugation with GC, they showed less photo-toxicity compared to PpIX- or Ce6-loaded GC NPs in an in vitro study. However, in vivo imaging studies showed that they had a prolonged circulation time and accumulated more specifically in the tumor, resulting in better therapeutic efficacy. For example, Ce6-conjugated GC NPs showed a dramatic decrease of tumor volume (about $160 \mathrm{~mm}^{3}$ ) at 20 day post-treatment, which was significantly smaller than that of Ce6-loaded GC NPs-treated mice (about $560 \mathrm{~mm}^{3}$ ) [55]. These results suggest that the factors of burst drug release and stability of NPs in vivo should be considered when constructing photoactivatable theranostic nanoagents for PDT.

The bioorthogonal chemical reporter strategy is a method for labeling and visualizing biomolecules in vivo without the requirement of genetic manipulation $[58,59]$. In this approach, metabolic labeling 
of the cell with azides primes the target biomolecule to be visualized by covalent attachment of an imaging probe. As a chemical reporter, azide is the most widely used because of its small size, metabolic stability, and lack of reactivity with natural biofunctionality [58,60]. The reaction of azide-alkyne cycloaddition forms an azide and a terminal alkyne (called "click chemistry"), which involves the use of a $\mathrm{Cu}$ catalyst [61,62]. Among various alkyne reagents, cyclooctynes react with azides without the use of copper, referred to as "Cu-free click chemistry," to achieve bioorthogonal labeling [63]. Copper-free click chemistry has been widely applied in biological and biomedical fields, such as in the labeling of proteins, nucleotides, or cells; in the analysis of metabolic pathways; and for the surface modification of nanoagents [64-67]. Recently, Lee et al. reported a novel two-step photoactivatable theranostic strategy in vivo via metabolic glycoengineering and $\mathrm{Cu}$-free click chemistry (Figure 5A) [68]. To achieve this approach, they prepared two different nanoagents as follows. Firstly, for generation of azide groups on tumor tissues, the precursor (tetraacetylated $N$-azidoacetyl-D-mannosamine, Ac4ManNAz) was loaded into GC NPs with an amphiphilic structure via hydrophobic interactions. When Ac4ManNAz-loaded GC NPs were added to tumor cells or tumor-bearing mice, azide groups were site-specifically generated on tumor cells or tissues by metabolic glycoengineering after effective delivery and rapid uptake of Ac4ManNAz-loaded GC NPs into tumor cells. An in vitro cell study showed that Ac4ManNAz-loaded GC NPs enabled a longer lifetime for the azide groups on cells compared to free Ac4ManNAz because of the sustained release of Ac 4 ManNAz from GC NPs (Figure 5B). Furthermore, Ac4ManNAz-loaded GC NPs successfully generated large amounts of azide groups irrespective of the kind of tumor cells targeted (KB, A549, U87MG, MCF7, MDA-MD-468, and MDA-MD-436). Immunohistochemistry and fluorescence images also demonstrated that Ac4ManNAz-loaded GC NPs with a longer circulation time and a higher tumor-targeting efficiency effectively delivered Ac4ManNAz, resulting in much greater generation of the azide group on tumor tissues compared to normal tissues (i.e., liver, lung, spleen, and kidney) (Figure 5C,D). Secondly, for specific delivery of photoactivatable agents to azide groups generated on tumor tissues by copper-free click chemistry, bicycle [6.1.0] nonyne (BCN)-PEG-NHS and Ce6 were conjugated with glycol chitosan polymer to obtain BCN-Ce6-GC NPs. Through copper-free click chemistry, BCN-Ce6-GC NPs specifically bind with azide groups generated on tumor cell membranes by Ac4ManNAz-loaded GC NPs. Compared to free Ce6 and Ce6-GC NPs, BCN-Ce6-GC NPs were more accumulated in tumor regions after azide generation caused by pretreatment with Ac4ManNAz-loaded GC NPs (Figure 5E). In vivo photodynamic therapy demonstrated that a two-step strategy using Ac4ManNAz-loaded GC NPs and BCN-Ce6-GC NPs showed significant black scab generation and effective tumor destruction after laser irradiation compared to use of free $\mathrm{Ce} 6$ or BCN-Ce6-GC NPs alone (Figure 5F). This two-step theranostic approach has great potential for the enhancement of the tumor-targeting ability of theranostic agents and their therapeutic effects in cancer therapy. However, further optimized conditions are required to more specifically generate azide groups on target-diseased sites. 
Figure 5. (A) Schematic illustration of the two-step in vivo tumor targeting strategy for nanoparticles via metabolic glycoengineering and click chemistry; (B) Time-dependent lifetime of azide groups generated by free Ac 4 ManNAz and Ac4ManNAz-loaded GC NPs; (C) Intravenous injection of Ac4ManNAz-loaded GC NPs and metabolic glycoengineering on tumor tissue in vivo; (D) Western blot analysis of major organs and tumor tissue after i.v. injection of AC4ManNAz-loaded GC NPs; (E) Tumor targeted image of BCN-Ce6-GC NPs in tumor bearing mice after pretreatment of Ac4ManNAz-GC NPs; (F) In vivo photodynamic therapy in tumor bearing mice and tumor images of mice treated with BCN-Ce6-GC NPs and Ac4ManNAz-GC NPs at day seven and 21. Reprinted with permission from [68], Copyright (C) 2014 American Chemical Society.

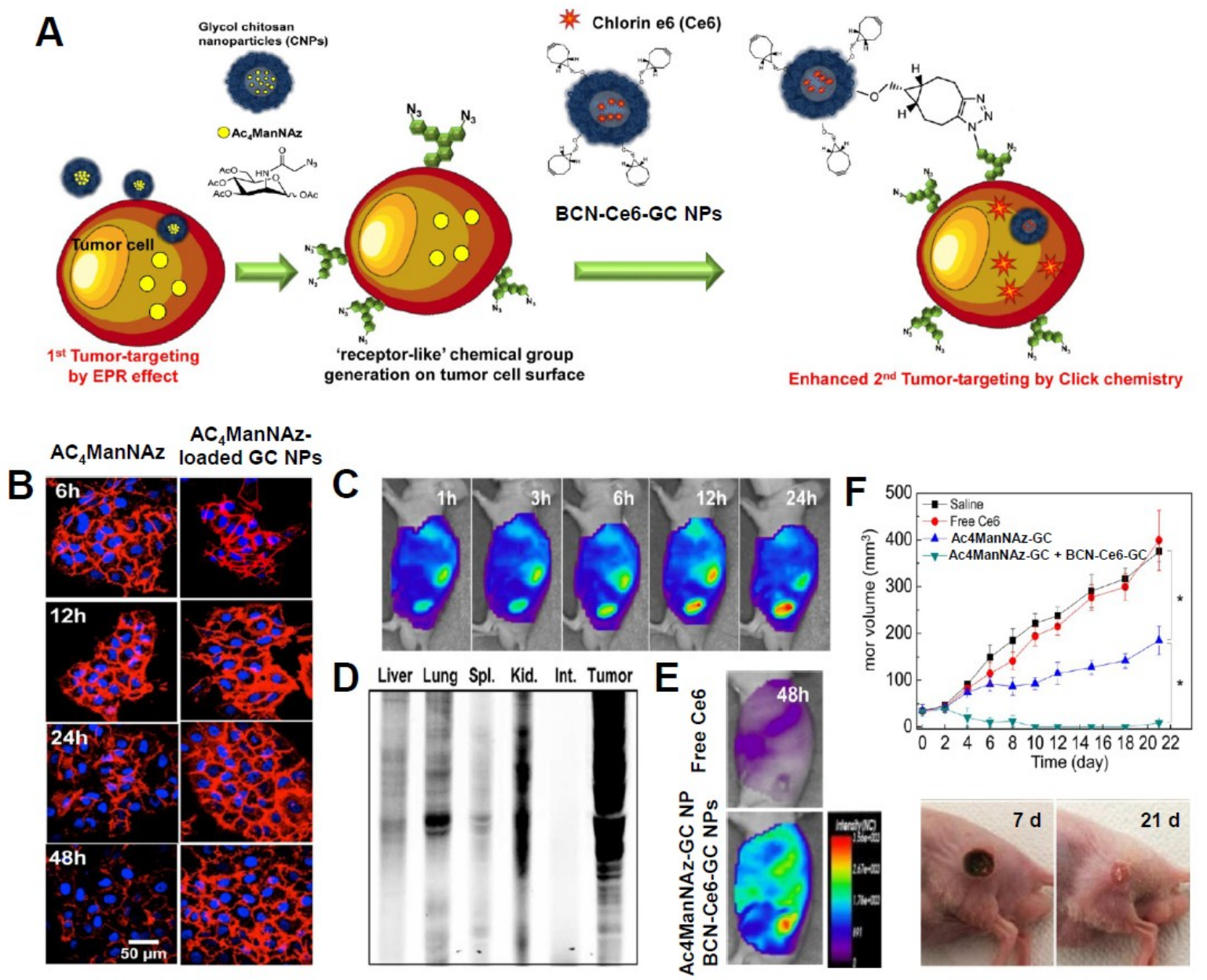

Tumor tissues have a more acidic microenvironment due to lactic acid production in hypoxic areas. Indeed, solid tumors with a $\mathrm{pH}$ ranging from 5.8 to 7.7 are on average 0.5 units lower than the $\mathrm{pH}$ of normal tissues. Thus, the use of different $\mathrm{pH}$ environments has been a promising avenue for cancer imaging and therapy. Recently, Park et al. developed a photoactivatible theranostic nanoagent that quickly switches into an aggressive molecule for tumor imaging and destruction within the acidic environment of the tumor [69]. The smart $\mathrm{pH}$-sensitive photoactivatable theranostic system consists of a GC backbone, a functional 3-diethylaminopropyl isothiocyanate (DEAP, pH sensitive moiety), a Ce6 block (photosensitizer), and polyethylene glycol (PEG). This theranostic nanoagent includes an intelligent switch from self-assembly (i.e., self-quenched state of photosensitizer) at physiological pH 7.4 
into extended random molecules (i.e., dequenched state for ROS production) at the extracellular acidic $\mathrm{pH}$. At physiological $\mathrm{pH} 7.4$, it forms a self-assembled molecule, $150 \mathrm{~nm}$ in diameter, and shows no singlet-oxygen production or noticeable cell death. However, when exposed to extracellular acidic $\mathrm{pH}$ ( $\mathrm{pH} 6.8$ or 6.4 ), it changes to the dequenched state, thereby emitting a strong NIRF signal and singlet-oxygen generation, resulting in higher phototoxicity and efficient tumor destruction of HeLa cells. This $\mathrm{pH}$-sensitive photoactivatable smart system enables targeted high-dose cancer therapy while ensuring the safety of normal tissues.

\subsection{Fullerene-Based Theranostic Nanoagents}

Fullerene $\left(\mathrm{C}_{60}\right)$ is a soccer ball-shaped structure with 12 pentagons (due to $\mathrm{C}_{5}-\mathrm{C}_{5}$ single bonds) and 20 hexagons $\left(\mathrm{C}_{5}-\mathrm{C}_{6}\right.$ double bonds). Since the discovery of $\mathrm{C}_{60}$ in 1985 , fullerene has attracted much attention and has been viewed as having great potential for a variety of applications. Due to its unique chemical structure, $\mathrm{C}_{60}$ possesses interesting photo-physical properties and generates ROS by exposure to visible light [70] and thereby can be used as a potentially strong photoactivatable agent for PDT in biological systems [44]. However, due to its inherent extreme hydrophobicity and innate tendency to aggregate in water and biological media, $\mathrm{C}_{60}$ has inefficient photoactivity and is less promising for application in photoactivatable drugs in biomedicine [44]. To overcome this shortcoming of $\mathrm{C}_{60}$, it was chemically conjugated to polysaccharides, such as GC and hyaluronic acid [71-73]. Unlike pristine $\mathrm{C}_{60}$ molecules, which rapidly aggregated within $5 \mathrm{~min}$, $\mathrm{GC}_{-} \mathrm{C}_{60}$ conjugates formed self-organized nanoparticles (approximately 10-23 nm in diameter) in PBS and were stable for more than one month without any precipitation [71]. Solubilization of $\mathrm{C}_{60}$ seemed to improve the light-sensitization of $\mathrm{C}_{60}$ molecules. When illuminated with a $670-\mathrm{nm}$ laser source, GC-C60 conjugates generated singlet oxygen and significantly induced $\mathrm{KB}$ cell death, whereas free $\mathrm{C}_{60}$ conjugates did not. More recently, Kim et al. developed an endosomal $\mathrm{pH}$-activated GC-fullerene derivative for PDT that was prepared using a simple two-step chemical reaction of (i) 2,3-dimethylmaleic acid (DMA) to free amine groups of GC and (ii) free hydroxyl groups of GC-DMA to $\pi$ - $\pi$ carbon bonds of $\mathrm{C}_{60}$ [73]. The GC-DMA-C60 conjugates formed self-assembled multi-nanogel aggregates $(283 \mathrm{~nm})$ at $\mathrm{pH} 7.4$ due to the electrostatic interactions between pendant DMA groups and the residual free amine groups of GC. During light illumination at $670 \mathrm{~nm}$, GC-DMA-C60 nanogels showed less generation of singlet oxygen and less cytotoxicity of $\mathrm{KB}$ cells at $\mathrm{pH} 7.4$ owing to an increased photo-interference effect between $\mathrm{C}_{60}$ molecules close packed in multi-nanogel aggregates. However, these multi-nanogel aggregates were divided into single nanogel parts (approximately $46 \mathrm{~nm}$ ) at endosomal $\mathrm{pH} 5.0$ due to the reduction of electrostatic interactions resulting from cleavage of the DMA blocks [74]. In particular, singlet oxygen generation and phototoxicity were significantly increased at endosomal $\mathrm{pH}$ 5.0. Another important consideration is that the solubilized GC-fullerene derivatives enable photo-luminescent tumor imaging without labeling of any fluorophores or isotopes [71-73]. Indeed, in vivo fluorescence images demonstrated that $\mathrm{GC}_{-} \mathrm{C}_{60}$ - or GC-DMA-C 60 -emitting fluorescence signals accumulated in tumor tissues in KB tumor-bearing mice. These solubilized fullerene derivatives are useful and promising for PDT. However, the fluorescence intensities of fullerene derivatives seem to be weaker than those of NIR fluorophores and other photosensitizers. These concerns should be further investigated to achieve high-resolution in fluorescence imaging. 


\section{Conclusions}

This review article discusses the recent progress of GC-based fluorescent theranostic nanoagents for simultaneous diagnosis and therapy in cancer treatments. Due to their biocompatible, biodegradable, and low-immunogenic properties, GC and its derivatives have been extensively studied for use in a wide range of biomedical applications. In particular, GC polymer has a large number of amine groups on its GC backbone and so can be covalently or non-covalently modified with hydrophobic molecules (bile acid analogs), drugs (chemotherapeutic small molecular drugs, nucleic acids, photosensitizers, or fullerenes), signal emitters (NIR fluorophores, photosensitizers, or fullerenes), and other imaging tracers (isotopes) in order to yield theranostic nanosystems. These GC-based fluorescent theranostic NPs showed good colloidal stability for extended circulation in the blood stream, excellent deformability to avoid in vivo filtration by the liver or spleen, and/or rapid cellular uptake characteristics to facilitate delivery of theranostic agents to target sites. Due to these features, GC-based theranostic nanosystems have excellent tumor-targeting characteristics, leading to effective therapeutic results. Therefore, GC-based theranostic nanosystems will play important roles in future biomedical applications and personalized medicine. In almost all works concerning chitosan applications, chitosan and its derivatives are non-toxic, biologically compatible materials, and thus suitable for the drug delivery carriers. Sometimes, the issue for their biocompatibilities is completely disregarded due to the statement on chitosan approval by the American Food and Drug Administration (FDA) as a wound dressing material [75]. However, their biocompatibility must be addressed and considered separately, requiring specific testing in the particular conditions expected for its administration because each particular case may be quite different in several different structures, formulation, and carriers in varied conditions [76]. The manufacturing processes also needed to meet the requirements for simultaneous diagnosis and therapy of theranostic nanosystems in a single platform are generally complicated. Due to the increased complexity, the technical challenges, such as cost, colloidal stability, and reproducibility, must be considered. Future improvements should be focused on the development of innovative strategies to overcome these technical challenges.

\section{Acknowledgments}

This research was supported in part by a Korea Basic Science Institute (KBSI) grant (T34525) to J.K. Rhee, another grant from the KBSI (D34400), and a grant of the National Research Foundation of Korea (NRF) funded by the Ministry of Education, and Science and Technology (No. 2012R1A2A2A04046108).

\section{Author Contributions}

K. Park and D. H. Yang conceived and designed the study. J.-K. Rhee, O.K. Park, and A. Lee collected the literature and analyzed the data. J.K. Rhee, O.K. Park, K. Park and D.H. Yang wrote the manuscript.

\section{Conflicts of Interest}

The authors declare no conflicts of interest. 


\section{References}

1. Sumer, B.; Gao, J. Theranostic nanomedicine for cancer. Nanomedicine 2008, 3, 137-140.

2. McCarthy, J.R. The future of theranostic nanoagents. Nanomedicine 2009, 4, 693-695.

3. McCarthy, J.R. Multifunctional agents for concurrent imaging and therapy in cardiovascular disease. Adv. Drug Deliv. Rev. 2010, 62, 1023-1030.

4. Fang, C.; Zhang, M. Nanoparticle-based theragnostics: Integrating diagnostic and therapeutic potentials in nanomedicine. J. Control. Release 2010, 146, 2-5.

5. Ahmed, N.; Fessi, H.; Elaissari, A. Theranostic applications of nanoparticles in cancer. Drug Discov. Today 2012, 17, 928-934.

6. Muthu, M.S.; Leong, D.T.; Mei, L.; Feng, S.S. Nanotheranostics-Application and further development of nanomedicine strategies for advanced theranostics. Theranostics 2014, 4, 660-677.

7. Maeda, H.; Wu, J.; Sawa, T.; Matsumura, Y.; Hori, K. Tumor vascular permeability and the EPR effect in macromolecular therapeutics: A review. J. Control. Release 2000, 65, 271-284.

8. Allen, T.M.; Cullis, P.R. Drug delivery systems: Entering the mainstream. Science 2004, 303, $1818-1822$.

9. Emerich, D.F.; Thanos, C.G. The pinpoint promise of nanoparticle-based drug delivery and molecular diagnosis. Biomol. Eng. 2006, 23, 171-184.

10. Koo, Y.E.; Reddy, G.R.; Bhojani, M.; Schneider, R.; Philbert, M.A.; Rehemtulla, A.; Ross, B.D.; Kopelman, R. Brain cancer diagnosis and therapy with nanoplatforms. Adv. Drug Deliv. Rev. 2006, 58, 1556-1577.

11. Weissleder, R. Molecular imaging in cancer. Science 2006, 312, 1168-1171.

12. Weissleder, R.; Pittet, M.J. Imaging in the era of molecular oncology. Nature 2008, 452, 580-589.

13. Lee, D.E.; Koo, H.; Sun, I.C.; Ryu, J.H.; Kim, K.; Kwon, I.C. Multifunctional nanoparticles for multimodal imaging and theragnosis. Chem. Soc. Rev. 2012, 41, 2656-2672.

14. Chen, X.; Conti, P.S.; Moats, R.A. In vivo near-infrared fluorescence imaging of integrin alphavbeta3 in brain tumor xenografts. Cancer Res. 2004, 64, 8009-8014.

15. Weissleder, R. A clearer vision for in vivo imaging. Nat. Biotechnol. 2001, 19, 316-317.

16. Shukla, S.K.; Mishra, A.K.; Arotiba, O.A.; Mamba, B.B. Chitosan-based nanomaterials: A state-of-the-art review. Int. J. Biol. Macromol. 2013, 59, 46-58.

17. Son, Y.J.; Jang, J.S.; Cho, Y.W.; Chung, H.; Park, R.W.; Kwon, I.C.; Kim, I.S.; Park, J.Y.; Seo, S.B.; Park, C.R.; et al. Biodistribution and anti-tumor efficacy of doxorubicin loaded glycol-chitosan nanoaggregates by EPR effect. J. Control. Release 2003, 91, 135-145.

18. Hyung Park, J.; Kwon, S.; Lee, M.; Chung, H.; Kim, J.H.; Kim, Y.S.; Park, R.W.; Kim, I.S.; Bong Seo, S.; Kwon, I.C.; et al. Self-assembled nanoparticles based on glycol chitosan bearing hydrophobic moieties as carriers for doxorubicin: In vivo biodistribution and anti-tumor activity. Biomaterials 2006, 27, 119-126.

19. Kim, J.H.; Kim, Y.S.; Kim, S.; Park, J.H.; Kim, K.; Choi, K.; Chung, H.; Jeong, S.Y.; Park, R.W.; Kim, I.S.; et al. Hydrophobically modified glycol chitosan nanoparticles as carriers for paclitaxel. J. Control. Release 2006, 111, 228-234. 
20. Park, J.H.; Kwon, S.; Nam, J.O.; Park, R.W.; Chung, H.; Seo, S.B.; Kim, I.S.; Kwon, I.C.; Jeong, S.Y. Self-assembled nanoparticles based on glycol chitosan bearing 5beta-cholanic acid for RGD peptide delivery. J. Control. Release 2004, 95, 579-588.

21. Kim, J.H.; Kim, Y.S.; Park, K.; Kang, E.; Lee, S.; Nam, H.Y.; Kim, K.; Park, J.H.; Chi, D.Y.; Park, R.W.; et al. Self-assembled glycol chitosan nanoparticles for the sustained and prolonged delivery of antiangiogenic small peptide drugs in cancer therapy. Biomaterials 2008, 29, 1920-1930.

22. Yoo, H.S.; Lee, J.E.; Chung, H.; Kwon, I.C.; Jeong, S.Y. Self-assembled nanoparticles containing hydrophobically modified glycol chitosan for gene delivery. J. Control. Release 2005, 103, 235-243.

23. Huh, M.S.; Lee, S.Y.; Park, S.; Lee, S.; Chung, H.; Lee, S.; Choi, Y.; Oh, Y.K.; Park, J.H.; Jeong, S.Y.; et al. Tumor-homing glycol chitosan/polyethylenimine nanoparticles for the systemic delivery of siRNA in tumor-bearing mice. J. Control. Release 2010, 144, 134-143.

24. Park, K.; Kim, J.H.; Nam, Y.S.; Lee, S.; Nam, H.Y.; Kim, K.; Park, J.H.; Kim, I.S.; Choi, K.; Kim, S.Y.; et al. Effect of polymer molecular weight on the tumor targeting characteristics of self-assembled glycol chitosan nanoparticles. J. Control. Release 2007, 122, 305-314.

25. Lee, S.; Ryu, J.H.; Park, K.; Lee, A.; Lee, S.Y.; Youn, I.C.; Ahn, C.H.; Yoon, S.M.; Myung, S.J.; Moon, D.H.; et al. Polymeric nanoparticle-based activatable near-infrared nanosensor for protease determination in vivo. Nano Lett. 2009, 9, 4412-4416.

26. Nam, T.; Park, S.; Lee, S.Y.; Park, K.; Choi, K.; Song, I.C.; Han, M.H.; Leary, J.J.; Yuk, S.A.; Kwon, I.C.; et al. Tumor targeting chitosan nanoparticles for dual-modality optical/MR cancer imaging. Bioconjugate Chem. 2010, 21, 578-582.

27. Veiseh, O.; Gunn, J.W.; Zhang, M. Design and fabrication of magnetic nanoparticles for targeted drug delivery and imaging. Adv. Drug Deliv. Rev. 2010, 62, 284-304.

28. Lewandrowski, K.U.; Gresser, J.D.; Wise, D.L.; Trantol, D.J. Bioresorbable bone graft substitutes of different osteoconductivities: A histologic evaluation of osteointegration of poly(propylene glycol-co-fumaric acid)-based cement implants in rats. Biomaterials 2000, 21, 757-764.

29. Bamrungsap, S.; Zhao, Z.; Chen, T.; Wang, L.; Li, C.; Fu, T.; Tan, W. Nanotechnology in therapeutics: A focus on nanoparticles as a drug delivery system. Nanomedicine 2012, 7 , $1253-1271$.

30. Zhang, Y.; Zhang, M. Calcium phosphate/chitosan composite scaffolds for controlled in vitro antibiotic drug release. J. Biomed. Mater. Res. 2002, 62, 378-386.

31. Na, J.H.; Koo, H.; Lee, S.; Min, K.H.; Park, K.; Yoo, H.; Lee, S.H.; Park, J.H.; Kwon, I.C.; Jeong, S.Y.; Kim, K. Real-time and non-invasive optical imaging of tumor-targeting glycol chitosan nanoparticles in various tumor models. Biomaterials 2011, 32, 5252-5261.

32. Barshtein, G.; Ben-Ami, R.; Yedgar, S. Role of red blood cell flow behavior in hemodynamics and hemostasis. Expert Rev. Cardiovasc. Ther. 2007, 5, 743-752.

33. Kim, K.; Kim, J.H.; Park, H.; Kim, Y.S.; Park, K.; Nam, H.; Lee, S.; Park, J.H.; Park, R.W.; Kim, I.S.; et al. Tumor-homing multifunctional nanoparticles for cancer theragnosis: Simultaneous diagnosis, drug delivery, and therapeutic monitoring. J. Control. Release 2010, 146, 219-227. 
34. Saravanakumar, G.; Min, K.H.; Min, D.S.; Kim, A.Y.; Lee, C.M.; Cho, Y.W.; Lee, S.C.; Kim, K.; Jeong, S.Y.; Park, K.; et al. Hydrotropic oligomer-conjugated glycol chitosan as a carrier of paclitaxel: Synthesis, characterization, and in vivo biodistribution. J. Control. Release 2009, 140, $210-217$.

35. Kim, J.H.; Kim, Y.S.; Park, K.; Lee, S.; Nam, H.Y.; Min, K.H.; Jo, H.G.; Park, J.H.; Choi, K.; Jeong, S.Y.; et al. Antitumor efficacy of cisplatin-loaded glycol chitosan nanoparticles in tumor-bearing mice. J. Control. Release 2008, 127, 41-49.

36. Min, K.H.; Park, K.; Kim, Y.S.; Bae, S.M.; Lee, S.; Jo, H.G.; Park, R.W.; Kim, I.S.; Jeong, S.Y.; Kim, K.; et al. Hydrophobically modified glycol chitosan nanoparticles-encapsulated camptothecin enhance the drug stability and tumor targeting in cancer therapy. J. Control. Release 2008, 127, 208-218.

37. Hwang, H.Y.; Kim, I.S.; Kwon, I.C.; Kim, Y.H. Tumor targetability and antitumor effect of docetaxel-loaded hydrophobically modified glycol chitosan nanoparticles. J. Control. Release 2008, 128, 23-31.

38. Rasool, A.A.; Hussain, A.A.; Dittert, L.W. Solubility enhancement of some water-insoluble drugs in the presence of nicotinamide and related compounds. J. Pharm. Sci. 1991, 80, 387-393.

39. Lee, B.S.; Park, K.; Park, S.; Kim, G.C.; Kim, H.J.; Lee, S.; Kil, H.; Oh, S.J.; Chi, D.; Kim, K.; et al. Tumor targeting efficiency of bare nanoparticles does not mean the efficacy of loaded anticancer drugs: Importance of radionuclide imaging for optimization of highly selective tumor targeting polymeric nanoparticles with or without drug. J. Control. Release 2010, 147, 253-260.

40. Kelkar, S.S.; Reineke, T.M. Theranostics: Combining imaging and therapy. Bioconjugate Chem. 2011, 22, 1879-1903.

41. Niidome, T.; Huang, L. Gene therapy progress and prospects: Nonviral vectors. Gene Ther. 2002, 9, 1647-1652.

42. Lee, S.Y.; Huh, M.S.; Lee, S.; Lee, S.J.; Chung, H.; Park, J.H.; Oh, Y.K.; Choi, K.; Kim, K.; Kwon, I.C. Stability and cellular uptake of polymerized siRNA (poly-siRNA)/polyethylenimine (PEI) complexes for efficient gene silencing. J. Control. Release 2010, 141, 339-346.

43. Lee, S.J.; Huh, M.S.; Lee, S.Y.; Min, S.; Lee, S.; Koo, H.; Chu, J.U.; Lee, K.E.; Jeon, H.; Choi, Y.; et al. Tumor-homing poly-siRNA/glycol chitosan self-cross-linked nanoparticles for systemic siRNA delivery in cancer treatment. Angew. Chem. 2012, 51, 7203-7207.

44. Sharma, S.K.; Chiang, L.Y.; Hamblin, M.R. Photodynamic therapy with fullerenes in vivo: Reality or a dream? Nanomedicine 2011, 6, 1813-1825.

45. Agostinis, P.; Berg, K.; Cengel, K.A.; Foster, T.H.; Girotti, A.W.; Gollnick, S.O.; Hahn, S.M.; Hamblin, M.R.; Juzeniene, A.; Kessel, D.; et al. Photodynamic therapy of cancer: An update. Cancer J. Clin. 2011, 61, 250-281.

46. Dolmans, D.E.; Fukumura, D.; Jain, R.K. Photodynamic therapy for cancer. Nat. Rev. Cancer 2003, 3, 380-387.

47. Henderson, B.W.; Dougherty, T.J. How does photodynamic therapy work? Photochem. Photobiol. 1992, 55, 145-157.

48. Josefsen, L.B.; Boyle, R.W. Unique diagnostic and therapeutic roles of porphyrins and phthalocyanines in photodynamic therapy, imaging and theranostics. Theranostics 2012, 2, 916-966. 
49. Moore, C.M.; Pendse, D.; Emberton, M. Photodynamic therapy for prostate cancer-A review of current status and future promise. Nat. Clin. Pract. Urol. 2009, 6, 18-30.

50. Awan, M.A.; Tarin, S.A. Review of photodynamic therapy. Surgeon 2006, 4, 231-236.

51. Sun, Y.; Chen, Z.L.; Yang, X.X.; Huang, P.; Zhou, X.P.; Du, X.X. Magnetic chitosan nanoparticles as a drug delivery system for targeting photodynamic therapy. Nanotechnology 2009, 20, 135102.

52. Guillemard, V.; Saragovi, H.U. Taxane-antibody conjugates afford potent cytotoxicity, enhanced solubility, and tumor target selectivity. Cancer Res. 2001, 61, 694-699.

53. Bechet, D.; Couleaud, P.; Frochot, C.; Viriot, M.L.; Guillemin, F.; Barberi-Heyob, M. Nanoparticles as vehicles for delivery of photodynamic therapy agents. Trends Biotechnol. 2008, 26, 612-621.

54. Lee, S.J.; Park, K.; Oh, Y.K.; Kwon, S.H.; Her, S.; Kim, I.S.; Choi, K.; Lee, S.J.; Kim, H.; Lee, S.G.; et al. Tumor specificity and therapeutic efficacy of photosensitizer-encapsulated glycol chitosan-based nanoparticles in tumor-bearing mice. Biomaterials 2009, 30, 2929-2939.

55. Lee, S.J.; Koo, H.; Jeong, H.; Huh, M.S.; Choi, Y.; Jeong, S.Y.; Byun, Y.; Choi, K.; Kim, K.; Kwon, I.C. Comparative study of photosensitizer loaded and conjugated glycol chitosan nanoparticles for cancer therapy. J. Control. Release 2011, 152, 21-29.

56. Bae, Y.H.; Yin, H. Stability issues of polymeric micelles. J. Control. Release 2008, 131, $2-4$.

57. Lee, S.J.; Koo, H.; Lee, D.E.; Min, S.; Lee, S.; Chen, X.; Choi, Y.; Leary, J.F.; Park, K.; Jeong, S.Y.; et al. Tumor-homing photosensitizer-conjugated glycol chitosan nanoparticles for synchronous photodynamic imaging and therapy based on cellular on/off system. Biomaterials 2011, 32, 4021-4029.

58. Baskin, J.M.; Prescher, J.A.; Laughlin, S.T.; Agard, N.J.; Chang, P.V.; Miller, I.A.; Lo, A.; Codelli, J.A.; Bertozzi, C.R. Copper-free click chemistry for dynamic in vivo imaging. Proc. Natl. Acad. Sci. USA 2007, 104, 16793-16797.

59. Chang, P.V.; Prescher, J.A.; Sletten, E.M.; Baskin, J.M.; Miller, I.A.; Agard, N.J.; Lo, A.; Bertozzi, C.R. Copper-free click chemistry in living animals. Proc. Natl. Acad. Sci. USA 2010, 107, 1821-1826.

60. Prescher, J.A.; Bertozzi, C.R. Chemistry in living systems. Nat. Chem. Biol. 2005, 1, 13-21.

61. Rostovtsev, V.V.; Green, L.G.; Fokin, V.V.; Sharpless, K.B. A stepwise huisgen cycloaddition process: Copper(I)-catalyzed regioselective "ligation" of azides and terminal alkynes. Angew. Chem. 2002, 41, 2596-2599.

62. Tornoe, C.W.; Christensen, C.; Meldal, M. Peptidotriazoles on solid phase: [1,2,3]-triazoles by regiospecific copper(i)-catalyzed 1,3-dipolar cycloadditions of terminal alkynes to azides. J. Org. Chem. 2002, 67, 3057-3064.

63. Agard, N.J.; Prescher, J.A.; Bertozzi, C.R. A strain-promoted [3 + 2] azide-alkyne cycloaddition for covalent modification of biomolecules in living systems. J. Am. Chem. Soc. 2004, 126, $15046-15047$.

64. Ngo, J.T.; Champion, J.A.; Mahdavi, A.; Tanrikulu, I.C.; Beatty, K.E.; Connor, R.E.; Yoo, T.H.; Dieterich, D.C.; Schuman, E.M.; Tirrell, D.A. Cell-selective metabolic labeling of proteins. Nat. Chem. Biol. 2009, 5, 715-717. 
65. Cheng, Z.; Al Zaki, A.; Hui, J.Z.; Muzykantov, V.R.; Tsourkas, A. Multifunctional nanoparticles: Cost versus benefit of adding targeting and imaging capabilities. Science 2012, 338, 903-910.

66. Gartner, Z.J.; Bertozzi, C.R. Programmed assembly of 3-dimensional microtissues with defined cellular connectivity. Proc. Natl. Acad. Sci. USA 2009, 106, 4606-4610.

67. Bertozzi, C.R.; Kiessling, L.L. Chemical glycobiology. Science 2001, 291, 2357-2364.

68. Lee, S.; Koo, H.; Na, J.H.; Han, S.J.; Min, H.S.; Lee, S.J.; Kim, S.H.; Yun, S.H.; Jeong, S.Y.; Kwon, I.C.; et al. Chemical tumor-targeting of nanoparticles based on metabolic glycoengineering and click chemistry. ACS Nano 2014, 8, 2048-2063.

69. Park, S.Y.; Baik, H.J.; Oh, Y.T.; Oh, K.T.; Youn, Y.S.; Lee, E.S. A smart polysaccharide/drug conjugate for photodynamic therapy. Angew. Chem. 2011, 50, 1644-1647.

70. Jensen, A.W.; Wilson, S.R.; Schuster, D.I. Biological applications of fullerenes. Bioorg. Med. Chem. 1996, 4, 767-779.

71. Kwag, D.S.; Oh, N.M.; Oh, Y.T.; Oh, K.T.; Youn, Y.S.; Lee, E.S. Photodynamic therapy using glycol chitosan grafted fullerenes. Int. J. Pharm. 2012, 431, 204-209.

72. Kwag, D.S.; Park, K.; Oh, K.T.; Lee, E.S. Hyaluronated fullerenes with photoluminescent and antitumoral activity. Chem. Commun. 2013, 49, 282-284.

73. Kim, S.; Lee, D.J.; Kwag, D.S.; Lee, U.Y.; Youn, Y.S.; Lee, E.S. Acid pH-activated glycol chitosan/fullerene nanogels for efficient tumor therapy. Carbohydr. Polym. 2014, 101, 692-698.

74. Lee, B.R.; Oh, K.T.; Baik, H.J.; Youn, Y.S.; Lee, E.S. A charge-switched nano-sized polymeric carrier for protein delivery. Int. J. Pharm. 2010, 392, 78-82.

75. Kean, T.; Thanou, M. Biodegradation, biodistribution and toxicity of chitosan. Adv. Drug Deliv. Rev. 2010, 62, 3-11.

76. Rodrigues, S.; Dionísio, M.; López, C.R.; Grenha, A. Biocompatibility of chitosan carriers with application in drug delivery. J. Funct. Biomater. 2012, 3, 615-641.

(C) 2014 by the authors; licensee MDPI, Basel, Switzerland. This article is an open access article distributed under the terms and conditions of the Creative Commons Attribution license (http://creativecommons.org/licenses/by/4.0/). 